\title{
Mathematical Tables on Punched Cards
}

The increasing number of scientific computing laboratories employing the punched card method in general scientific calculation indicates the need for more general dissemination of information concerning mathematical tables on punched cards. In the past when the number of such laboratories was small, the exchange of information was made by personal contact and informal correspondence. It is felt that a wider distribution of this information will not only facilitate the exchange of card files between laboratories but may, on occasion, make it possible for a scientist without punched card facilities to utilize a printed list of selected data from the cards at one of the laboratories.

One of the functions of the new Watson Scientific Computing Laboratory at Columbia University will be to maintain a card file of current information concerning mathematical tables on punched cards at the various laboratories. This information will be available by correspondence, and such portions as are suitable may be published from time to time in $M T A C .^{1}$

The question of how much of this detailed material should be published is difficult and can probably be answered only by experience; the comments of the readers will, no doubt, influence future publication. Owing to the ease of constructing and re-arranging tabular data on punched cards, the tables tend to be more fluid in nature than printed tables and to reflect not only the problem for which they were constructed but the particular equipment which was available. These and other factors affect the problem of maintaining a current detailed printed description of such tables.

The following list of tables has been prepared from material on file in World Headquarters of the International Business Machines Corporation. The list is not complete, but it indicates some of the laboratories and the types of tables in use. Many laboratories are, of course, engaged in secret war work and it would be impossible now to give a complete list of the tables in use or even of the laboratories themselves.

ABERDEEN PROVING GROUND, BALLISTIC RESEARCH LABORATORY

\begin{tabular}{rll} 
File & \multicolumn{1}{c}{ Function and Accuracy } & \multicolumn{1}{c}{ Range and Interval } \\
1 & $\tan ^{-1} x$ in degrees (2D) & $0(.001) 3.75(.01) 18.25$ \\
2 & $\sin x(5 \mathrm{D}), \cos x(5 \mathrm{D}), \tan x(5 \mathrm{D})$ & $0(0.01) 45^{\circ}$ \\
3 & $\int_{0}^{x} \sqrt{1+x^{2}} d x(6 \mathrm{D})$ & $0(.1) 20$ \\
24 & $\sqrt{x}(8 \mathrm{D})$ & $.0001(.0001) 1(.001) 10$ \\
5 & $x^{2}, x^{3}, x^{4}, x^{5}$ & $0(.0001) 1$ \\
26 & $1 / x(6 \mathrm{D})$ & $.1(.0001) 1$ \\
7 & $1 / x(8 \mathrm{D})$ & $.001(.001) 1$ \\
8 & $\sqrt[3]{x}(7 \mathrm{D})$ & $1(.001) 10$ \\
29 & $\log x(7 \mathrm{D})$ & $1(.0001) 10$ \\
10 & Bessel functions $e^{-x} I_{0}(x)$, & \\
& $e^{-x} I_{1}(x), e^{-x} K_{0}(x), e^{-x} K_{1}(x)$ & $0(.02) 10$
\end{tabular}
$B=\frac{1}{2} \Delta^{2} i_{-1}$.

${ }^{1}$ For discussion connected with punched-card tables, see $M T A C$, p. $173 f, 334$.

2 These tables are provided with second order interpolation coefficients $A=\frac{1}{2}\left(\Delta_{i-1}+\Delta_{i}\right)$, 
11 Numerous extensive ballistic tables

12 Lagrangean interpolation coefficients

3 point (5D)

$0(.001) 1$

5 point $(7 \mathrm{D})$

$0(.001) 2$

See also $M T A C$, p. 332.

ARTHUR D. LITTLE, INC., CAMBRIDGE, MASS.

\section{CALIFORNIA INSTITUTE OF TECHNOLOGY, GATES \& CRELLIN}

\section{LABORATORIES OF CHEMISTRY}

File

1

Function and Accuracy

$A \sin 2 \pi h x, A \cos 2 \pi h x$
Range and Interval

$$
\begin{aligned}
x= & 0(.002) .25 \\
h= & 0(1) 30 \\
A= & \pm 1(1) 5 ; 10(10) 50 ; 100, \\
& 200 ; 500 .
\end{aligned}
$$

Accuracy (.01) except for $A= \pm 100(.005)$ and $\pm 500(.025)$

$2 A \sin 2 \pi h x$

$$
\begin{aligned}
x & =0(.05) 7.15 \\
h= & 0(.01) 5 \\
A= & \text { same as } 1 \text { with addition } \\
& \text { of }, \pm 300, \pm 400 .
\end{aligned}
$$

COLUMBIA UNIVERSITY, THE THOMAS J. WATSON ASTRONOMICAL COMPUTING BUREAU

File

1

2

3

4

5

6

7

File

1
Range and Interval $0\left(0^{\circ} .01\right) 90^{\circ}$ $0\left(10^{\prime \prime}\right) 30^{\circ}$ $0\left(1^{s}\right) 20^{m}$ $0(0: 1) 20^{m}$ 2.0(.001)7.5 Comrie 7.5(.01)20 $1000(1) 9999$

$\quad$ Source
Peters
Brandenburg
Comrie
$\sin x$ (7D), $\cos x$ (7D), $\tan x$ (7D) $\tan x$ (7D) $\tan x(7 \mathrm{D}), \sec x(7 \mathrm{D})$ $\tan x(8 \mathrm{D}), \sec x(8 \mathrm{D})$ $x^{-3 / 2}(8 D)$

$1 / n(10 \mathrm{D}), n^{2}$ Numerous extensive astronomical tables.

\section{INTERNATIONAL BUSINESS MACHINES CORPORATION,} NEW YORK SERVICE BUREAU

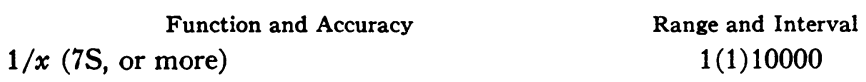

\section{MASSACHUSETTS INSTITUTE OF TECHNOLOGY, DEPARTMENT} OF ELECTRICAL ENGINEERING

Source

Peters

Peters

NYMTP

M.I.T.

M.I.T.

Barlow

Barlow

B. A. Tables VI 


\section{NATIONAL DEFENSE RESEARCH COMMITTEe, APPLIED MATHEMATICS PANEL}

\section{File Function and Accuracy}

$1 \log x(7 \mathrm{D})(5 \mathrm{D})$

\section{Range and Interval}

100(1)9999

\section{PRINCETON UNIVERSITY, NATIONAL DEFENSE RESEARCH COMMITTEE}

\begin{tabular}{clc} 
File & \multicolumn{1}{c}{ Function and Accuracy } & Range and Interval \\
1 & $\sqrt{x}(9 \mathrm{D})(6 \mathrm{D}), \sqrt{10 x}(8 \mathrm{D})(6 \mathrm{D}), 1 / x(7 \mathrm{D})(6 \mathrm{D})$, & \\
& $\Delta \sqrt{x}, \Delta \sqrt{10 x}, \Delta(1 / x)$ & $1(1) 9999$ \\
2 & $\log x(5 \mathrm{D}), \Delta \log x, 1 /(\Delta \log x), \log x /(\Delta \log x)$ & $1(1) 1000$ \\
3 & $\log \sin x(6 \mathrm{D}), \log \cos x(6 \mathrm{D}), \log \tan x(6 \mathrm{D})$, & $0(1) 800$ mils \\
& $\log \operatorname{ctn} x(6 \mathrm{D})$ & \\
4 & $1600-x, \sin x, \cos x, \tan x, \operatorname{ctn} x, \sec x, \csc x$, & \\
& $\frac{1}{2} \tan x, \frac{1}{2} \operatorname{ctn} x(\operatorname{all} 5 \mathrm{D}) ; \log \sin x, \log \cos x, 1600$ mils \\
& $\log \tan x . \operatorname{See} M T A C, \mathrm{p} \cdot 146$ & $0(.1) 160$
\end{tabular}

\section{UNIVERSITY OF MICHIGAN}

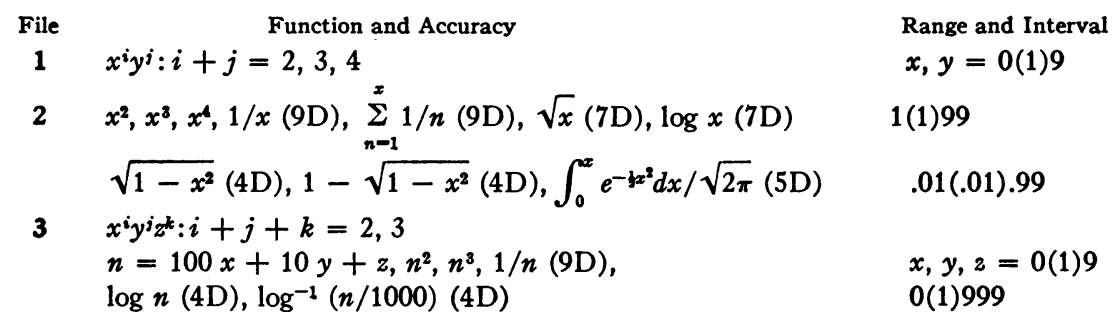

\section{U. S. LAKE SURVEY, MILITARY GRID UNIT}

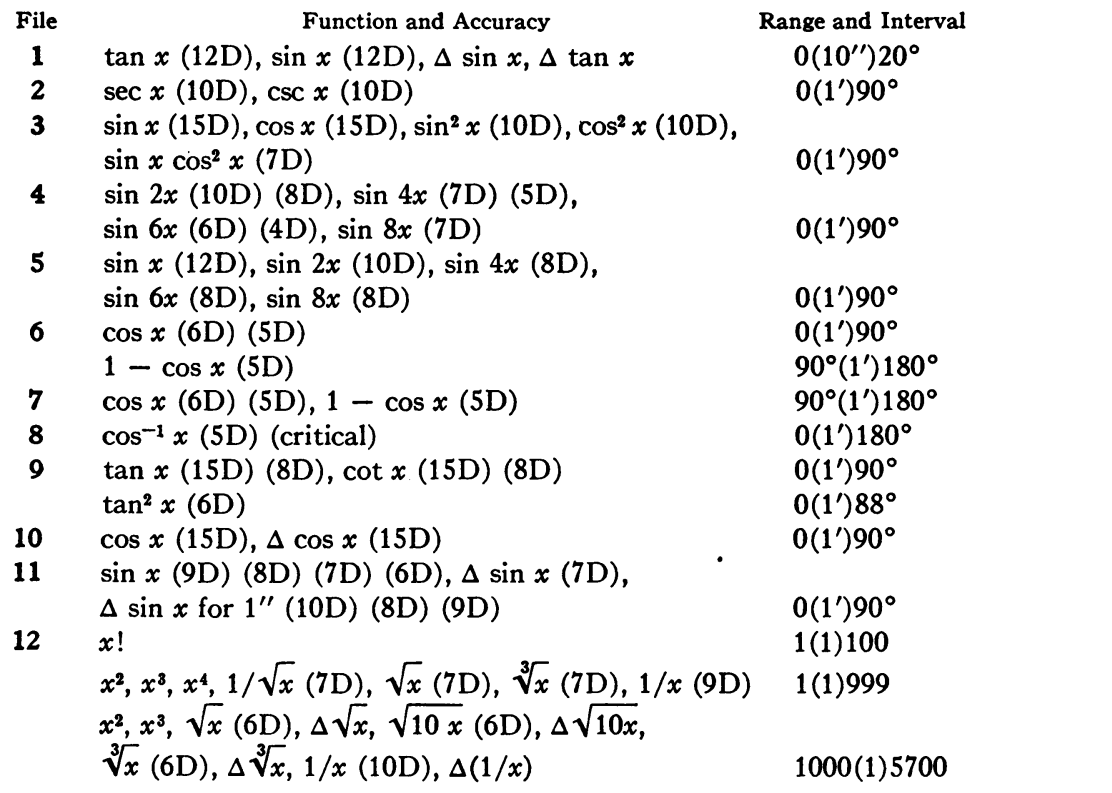

Spheroid functions (notation as in U.S.C. \& G.S.S.P. no. 8) for the following spheroids, Clarke 1880, Everest, Bessel, Hayford, Helmert, Airy, Clarke 1858, Plessis, Danish, Struve: 
13 meridional arc (.001 meters)

Range and Interval

$A(10 \mathrm{D}, 8 \mathrm{~S}), B(10 \mathrm{D}, 8 \mathrm{~S})$

$0\left(1^{\prime}\right) 90^{\circ}$

$C(15 \mathrm{D}, 7 \mathrm{~S}), D(12 \mathrm{D}, 4 \mathrm{~S})$,

$0\left(1^{\prime}\right) 90^{\circ}$

$N, R$ (both .001 meters)

$0\left(1^{\prime}\right) 90^{\circ}$

$0\left(1^{\prime}\right) 90^{\circ}$

16 military grid coordinates from U.S.C. \& G.S.S.P. no. 59

$X, Y$ (both .1 yard) Lat., Long. of U. S.

$5^{\prime}$ intersections

17 latitude transformation tables geometric-

isometric: isometric-geodetic for following

spheroids: Clarke 1866, 1880, 1860 ; Hayford,

Bessel, Everest

geodetic-authalic: authalic-geodetic for Clarke 1866

$0\left(1^{\prime}\right) 90^{\circ}$

$0\left(1^{\prime}\right) 90^{\circ}$

\section{U. S. NAVAL OBSERVATORY}

\begin{tabular}{clc} 
File & \multicolumn{1}{c}{ Function and Accuracy } & Range and Interval \\
1 & $\sin x(7 \mathrm{D}), \cos x(7 \mathrm{D}), \tan x(7 \mathrm{D})$ & $0(0: 01) 90^{\circ}$ \\
2 & $1 / x(6 \mathrm{D})$ & $1(.001) 4$ \\
& & $4(.01) 10$ \\
3 & $\log ^{-1} x(8 \mathrm{D})$ & $0(.001) 1$ \\
4 & $\operatorname{hav}^{-1} x($ about $0: 001)$ & 0 to $25^{\circ}$ \\
$\mathbf{5}$ & $\sin x(8 \mathrm{D}), \cos x(8 \mathrm{D})$ & $0(0: 01) 90^{\circ}$
\end{tabular}

Special tables for astronomical work.

VEGA AIRCRAFT CORPORATION

File

$1 \log x(8 \mathrm{D}), \Delta \log x(8 \mathrm{D}), 1 /(\Delta \log x)$

$10000(1) 100000$

W. J. E.

\section{RECENT MATHEMATICAL TABLES}

211[A].-W. VRIEsendorP, The Calculating Dictionary, Utrecht, L. E. Bosch \& Zoon, printer, for the author, 1937. [1003 p.], $13.5 \times 26.7 \mathrm{~cm} .17 .5$ Dutch florins.

This Dictionary contains the multiplications up to $999 \times 999$, except multiples of ten. There are two pages of examples showing applications of the volume in obtaining the products of larger numbers. On each page headed 2, 3, ., 999 are given 900 products. The Dutch edition has the title De Rekendictionnaire Tabellen bijeengebracht ten dienste van handel, industrie, administratie en onderwijs.

There is no reference to any other work offering the same tabular information, and readily available in 1937, such as: A. L. Crelle, Calculating Tables, Berlin, 1930 which has gone through many editions, since. the first two-volume edition in 1820 , including one by an insurance company in Japan (1913).

212[D].-M. J. Buerger \& Gilbert E. Klein, "Correction of X-ray intensities for Lorentz and polarization factors," Journal of Applied Physics, v. 16, July, 1945, p. 414-418. $20 \times 26.6 \mathrm{~cm}$.

Four-place tables are given for the following functions: $2 \sin 2 \theta /\left(1+\cos ^{2} 2 \theta\right)$ and $2 /\left(1+\cos ^{2} 2 \theta\right)$, for the argument $\sin \theta=0(.001) .999$. 